\title{
Impact induced damage beneath craters
}

\author{
Kaiwen Xia and Thomas J. Ahrens \\ Seismological Laboratory, California Institute of Technology , Pasadena, California 91125
}

\begin{abstract}
Ackermann et al. (1975) described the subsurface structure of Meteor Crater and identified a fractured rock zone extending to about $1 \mathrm{~km}$ deep. The depth of the fractured/damage zone can be used to extract information about the impact cratering process. We impacted rock samples (San Marcos gabbro) in the laboratory and imaged the damage structure using both dicing and tomography methods. We propose a simple model to describe the damage zone depth based on the laboratory measurements. The model agrees well with other methods for the estimation of the projectile size of Meteor Crater and it may be used for estimates of damage around craters of other planets and moons.
\end{abstract}

\section{Introduction}

The impact of asteroids and comets on planets leaves plenty of geological and geophysical features [Grieve and Pesonen, 1992]. Efforts have been put into constraining the cratering process by using these features to improve our understanding of the planetary records and the implications of impact cratering for planetary evolution. For example, studies of the volume of craters led to scaling laws to relate crater volume and impact parameters [Holsapple and Schmidt, 1982]. The damage zone beneath Meteor Crater was mapped by Ackermann using the seismic inverse method. Simmons et al (1973) pointed out that virtually the entire crust of the Moon down to the depth about 25 $\mathrm{km}$ suffered shock-induced crack damage. Following previous work [Hörz, 1969; Polanskey and Ahrens, 1990; Ahrens and Rubin, 1993; Liu and Ahrens, 1997], we performed impact cratering recovery experiments to study the damage zone due to projectile impact.

The damage zone is characterized by reduced $P$ and $S$ wave velocities due to the initiation and growth of cracks. The amount of damage can be defined as $D=1-\left(c_{D} / c_{0}\right)^{2}$ [Ahrens and Rubin, 1993], where $c_{D}$ is the $\mathrm{P}$ or $\mathrm{S}$ wave velocity of damaged rock and $c_{0}$ is the $P$ or $S$ velocity of undamaged rock. We applied two methods to obtain the $P$ wave velocity structure beneath laboratory craters: 1 ) cutting out a central plane from the recovered target underneath craters and dicing it into $1 \mathrm{~cm}^{3}$ cubes for ultrasonic measurements following the method used by Ahrens and Rubin [Ahrens and Rubin, 1993]; 2) using a mechanical source to generate a hemispherical ultrasonic wave and measuring the travel time for different rays going through the sample, which is used as the input for a tomography inversion. For the first method, we assumed that the additional damage due to cutting is negligible [Liu and Ahrens, 1997] and for the latter method, we assumed that rays go straight.

Copyright 2001 by the American Geophysical Union.

Paper number 2001GL013001.

0094-8276/01/2001GL013001\$05.00
Based on the measurements of the damage zone size, we developed a simple model following the commonly used attenuation relation for the peak shock pressure. The model gives a reasonable value for the critical damage pressure $P_{c}$ and attenuation index, $n$, for laboratory experiments. The application of the model to Meteor Crater implies that this simple model could be used for larger geological structures.

\section{Projectile impact experiments}

San Marcos gabbro targets in the laboratory were impacted by aluminum bullets launched by a .220 inch caliber rifle within the velocity range $0.8-1.2 \mathrm{~km} / \mathrm{s}$, which corresponds to a peak pressure range of 6-9 GPa. The peak pressure, $P_{0}$, is calculated using the impedance match method [Ahrens, 1987], knowing the impact velocity $v_{0}$ and the equation of state for both the target and the aluminum bullet. The size of the target is about $15 \mathrm{~cm}$. The dimension of the gabbro target is chosen such that within the pressure range of our experiments, the edge effect of the target can be neglected (i.e., the reflected wave from the boundary is weak enough not to induce damage), which is the case for planetary impact cratering. The damage distribution is rotationally symmetric about the normal impact axis, so we simplified our problem by studying only the damage distribution of one central plane along the impact axis.

\section{Damage structure imaging method}

\section{Dicing method}

Following the existing procedure [Polanskey and Ahrens, 1990 ], we cleaned the recovered target. We cut out a $1 \mathrm{~cm}$ thick central plate and then cut it into slabs along different angles to the impact axis. The slabs were then cut into cubes for ultrasonic test. We used a pair of ultrasonic transducers $(1 \mathrm{MHz})$ to measure the $\mathrm{P}$-wave velocity, one generates the ultrasonic signal and the other is used as a receiver. We plot P-wave measurements for one test with cubes along the impact axis in Figure 1. We can see that the damage decreases with the distance to the impact center. We define a damage depth, $L_{D}$, where the damage $D$ is about $0 . L_{D}$ is about $4.5 \mathrm{~cm}$ in this case.

\section{Tomography method}

Since the dicing method may introduce additional damage during the cutting process, we tried a none-damage method. We designed a mechanical ultrasonic source and determined the travel time for each ray using a pair of transducers. We used the DLS (damped least square) method to obtain the P-wave velocity structure for the central plane of the recovered rock sample.

Mechanical source The source is the impact of an aluminum ball (5/64 inch in diameter), which is launched by 


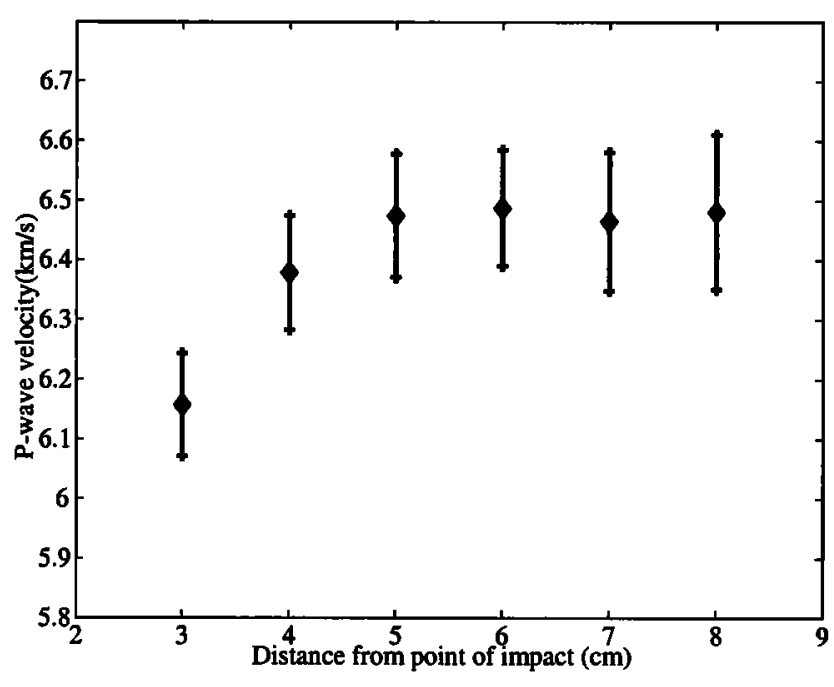

Figure 1. The $\mathrm{P}$-wave structure of the recovered sample along the impact axis obtained by dicing method. The error bar is obtained by the standard error propagation from uncertainties of the measurements of distance and travel time.

compressed gas with a velocity of about $3 \mathrm{~m} / \mathrm{s}$. The mechanical source has some advantages over transducer sources: 1 ) it generates a hemispherical ultrasonic wave instead of a beam-like wave by transducers so that we can measure the travel time for rays oblique to the surface; 2) the generated wave is so strong that it can penetrate the damage zone in therock sample; 3 ) the frequency of the wave can be easily tuned by changing the size and material of the impacting sphere. The peak energy of the mechanical source is located at a frequency about $0.5 \mathrm{MHz}$.

Travel time determination and inversion result We put a transducer next to the mechanical source (signal A) and another transducer at other places on the sample surface as a receiver (signal B). We plotted the signals in Figure 2. The travel time is determined by the time interval between the starting points of the signals assuming straight ray paths. The position of the mechanical source is $<1 \mathrm{~mm}$ from the position of transducer A, so we slightly underestimated the travel time. Since ray paths are longer

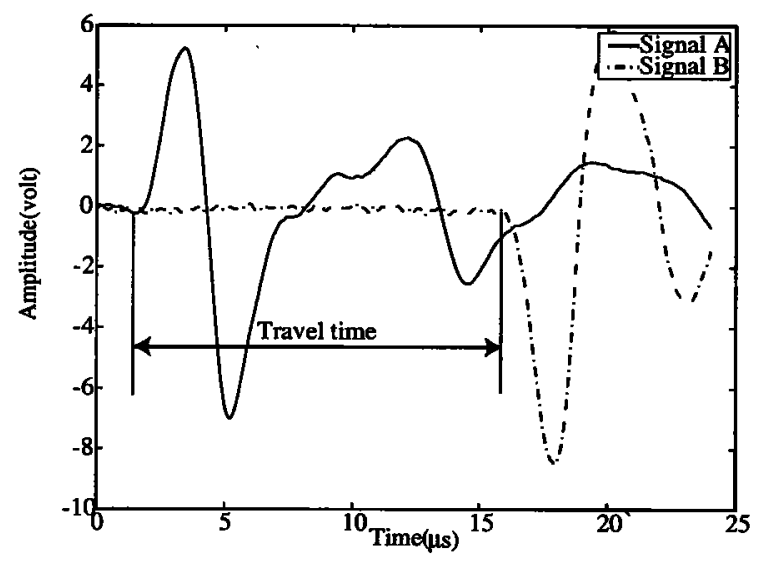

Figure 2. The determination of the travel time. We enlarged signal B 100 times to fit both signals in one picture.

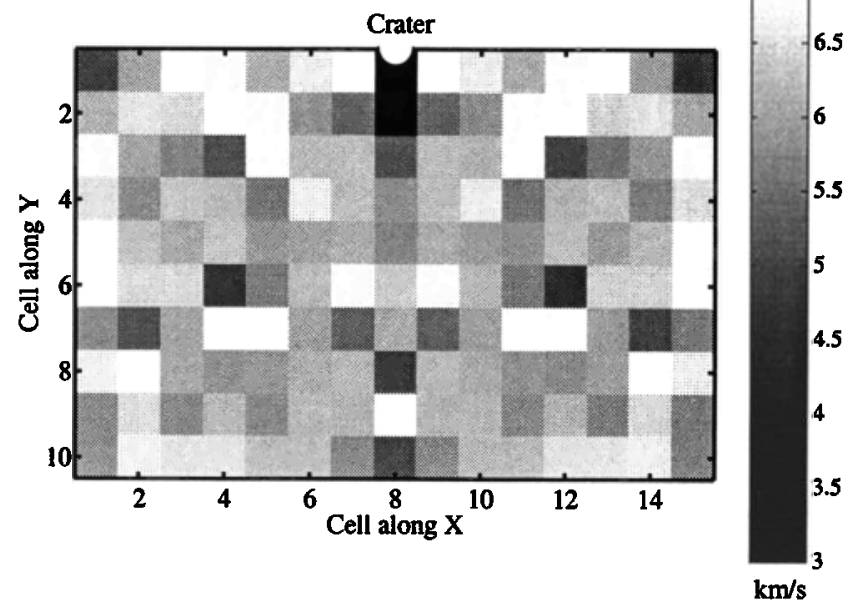

Figure 3. Results of the P-wave structure of the recovered rock by the damped least square method.

than $5 \mathrm{~cm}$, this error is within the error of the measurement. We divided the central plane $(10 \times 15 \mathrm{~cm})$ into 150 square cells $\left(1 \mathrm{~cm}^{2}\right)$ for the inversion. The result of the inversion is plotted in Figure 3. We can see damage zones around the crater. The damage depth $L_{D}$ is about $3.0 \mathrm{~cm}$, which is close to the damage depth obtained by the dicing method $(\sim 3.5 \mathrm{~cm})$ for a similar impact velocity. The random features outside the damage zone are due to errors in the measurements of travel time (finite size of transducer, bending rays, etc.). We think a better resolution can be obtained by denser sampling, but this would be very hard to achieve for the dicing method.

\section{Model for the maximum damage depth}

The impact of the projectile on the target generates a strong shock wave, which propagates into the target while decreasing in amplitude. The attenuation is usually described by, $P(r)=P_{0}(r / a)^{-n}$, where $a$ is the radius of the impactor ( $\sim 0.35 \mathrm{~cm}$ for the round-nose cylindrical bullet used in our experiments), $n$ is the attenuation index. We define $P_{c}$ as the critical pressure for damage, where:

$$
P_{c}=P_{0}\left(L_{D} / a\right)^{-n}
$$

Using this relation and the measurements of the damage depth $L_{D}$ and impact velocity, $v_{0}$, we determined by nonlinear fitting that for our laboratory experiments, as shown in Table $1, P_{c}=1.67 \pm 0.05 \mathrm{kbar}$ and $n=1.57 \pm 0.03$. For consistency, we used only the results from the dicing method. The value of $P_{c}$ is close to the spall strength, $P_{s}$, of the same rock, which is $1.44 \mathrm{kbar}$ [Lange et al., 1984]. This is expected since the furthest propagation damage is due to the extentional hoop stress within the shock front. The fracture pattern and mechanism of damage for projectile impact were addressed by Ahrens et al [Ahrens and Rubin, 1993].

Table 1. Measurements of Damage Depth

\begin{tabular}{cccc}
\hline Shot No. & 108 & 109 & 110 \\
\hline Impact velocity $/ \mathrm{km} / \mathrm{s}$ & 1.019 & 0.926 & 0.758 \\
Pressure/GPa & 9.2 & 7.6 & 6.2 \\
Damage Depth $/ \mathrm{cm}$ & $4.5 \pm 0.3$ & $4.0 \pm 0.3$ & $3.5 \pm 0.3$ \\
\hline
\end{tabular}




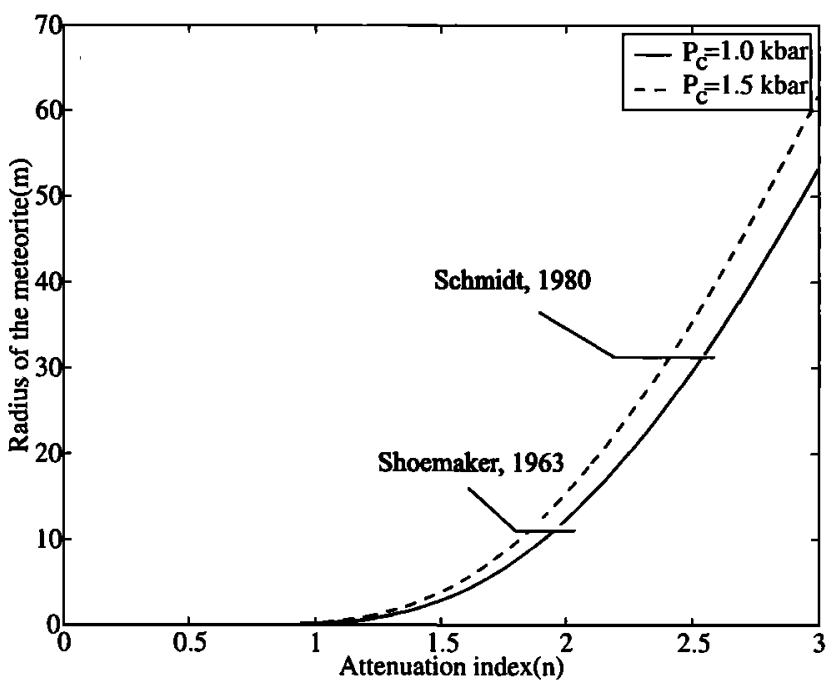

Figure 4. The radius of the meteorite as a function of the attenuation index, $n$, predicted by the model for two critical damage pressures.

We observed that the furthest propagating cracks are radial cracks, which are due to the extentional hoop stress. Theoretically, $n$ is between 1 (for elastic wave) and 3 (for plastic flow). So we have a reasonable value of $n$ for the current experimental pressure range. We can assume a depth dependent critical damage pressure underneath the surface of the Earth as:

$$
P_{c}=P_{s}+\rho g L_{D}
$$

where $\rho$ is the density of the overburden rocks. Thus we have a model for the damage depth for terrestrial craters.

\section{Application to the Meteor Crater}

Meteor Crater was formed about 50,000 years ago by the impact of an iron meteorite. The damage depth is about $1 \mathrm{~km}$ [Ackermann et al., 1975]. If we assume that the impact velocity is $20 \mathrm{~km} / \mathrm{s}$ [Schmidt, 1980], we can calculate the peak pressure on impact, $P_{0}$, with the equation of states of iron and rock found at Meteor Crater (Coconino sandstone). Using equation (1) and (2), we obtained an estimate of the radius of the meteorite as a function of the attenuation index, $n$. We used two critical damage pressures in our calculation, namely $1.0 \mathrm{kbar}$ and $1.5 \mathrm{kbar}$. The quasi-static extentional strength of sandstone under confining pressure about $0.1 \mathrm{kbar}$ is about $1.0 \mathrm{kbar}[$ Kirby and McCormick, 1989]. The strain rate effect will make the material stronger, so we calculated the case of critical damage pressure at $\mathbf{1 . 5}$ kbar. As we can see from Figure 4, since the attenuation index is $\sim 2$ for large scale hypersonic impact [Ahrens and O'Keefe, 1987], the radius predicted by this model is close to $12.4 \mathrm{~m}$ obtained by Shoemaker [1963] and $32.0 \mathrm{~m}$ by Schmidt [1980].

\section{Conclusions and Discussions}

1). The non-damage tomography method provides a suitable way to study the damage stucture of beneath laboratory craters in addition to the dicing method. The simple mechanical ultrasonic source suggested in this study offers one simple way to make the application possible.

2). The damage depth can be used as an independent constraint for the study of impact cratering and the preliminary model we described in this study could be possibly used for impact cratering problems of different scales.

Acknowledgments. This material is based upon work supported by the National Aeronautics and Space Administration under Grant No. NAG5-10198. Contribution number 8757, Division of Geological and Planetary Sciences, California Institute of Technology. The authors would like to thank Professor Ravichandran from Aeronautics, Caltech for insightful discussions. The authors would also like to thank the two reviewers for valuable comments.

\section{References}

Ackermann H. D., R. H. Godson and J. S. Watkins, A seismic refraction technique used for subsurface investigation at Meteor Crater, Arizona, J. Geophys. Res., 80,765-775, 1975.

Ahrens T. J., Shock wave techniques for geophysics and planetary physics, in Methods of Experimental Physics, edited by C. G. Sammis and T. L. Henyey, Vol. 24, pp. 185-235, Academic, San Diego, Calif., 1987.

Ahrens, T. J. and J. D. O'Keefe, Impact on the earth, ocean and atmosphere, Int. J. Impact Engng. 5, 13-32, 1987.

Ahrens T. J. and A. M. Rubin, Impact-induced tensinal failure in rock, J. Geophys. Res., 98, 1185-1203, 1993.

Grieve R. A. F. and L. J. Pesonen, The terrestrial impact cratering record, Tectonophysics, 216, 1-30, 1992.

Hörz, F., Structural and mineralogical evaluation of an experimentally produced impact crater in granite, Contr. Mineral. and Petrol. 21, 365-377, 1969.

Holsapple K. A. and R. M. Schmidt,On the scaling of crater dimensions 2. Impact processes, J. Geophys. Res., 87, 1849-1870, 1982.

Kirby H. K. and J. W. McCormick, Inelastic properties of rocks and minerals: strength and rheology, in CRC Practical Handbook of Physical Properties of Rocks and Minerals, edited by R. S. Carmichael, CRC Press, Inc., Boca Raton, Florida, 1989. Lange, M. A., T. J. Ahrens and M. B. Boslough, Impact cratering and spall failure of gabbro, Icarus 58, 383-395, 1984

Liu, C. and T. J. Ahrens, Stress wave attenuation in shockdamaged rock, J. Geophys. Res., 102, 5243-5250, 1997.

Polansky C. and T. J. Ahrens,Impact spallation experiments: Fracture patterns and spall velocities, Icarus 87, 140-155, 1990.

Schmidt R. M., Meteor Crater: Energy of formation-implication of centrifuge scaling, in Proc. Lunar Planet. Sci. Conf., 11, edited by Merrill R. B., pp. 2099-2128, Pergamon Press, 1980.

Shoemaker E. M., Impact mechanics at Meteor Crater, Arizona, in The solar system: Vol 4., The moon, meteorites, and comets, edited by Middlehurst B. M. and G. P. Kuiper, pp. 301-336, Chicago, University of Chicago Press, 1963.

Simmons G., T. Todd and H. Wang, The $25 \mathrm{~km}$ discontinuity: implication for lunar history, Science, 182, 158-161, 1973.

T. J. Ahrens and K. Xia, 252-21, Seismological Laboratory, California Institute of Technology, Pasadena, CA, 91125 (e-mail: xia@gps.caltech.edu)

(Received Feburary 13 2001; revised May 17, 2001; accepted June 27, 2001.) 